\title{
ADAPTIVE APPROACH FOR BLIND SOURCE SEPARATION OF NONLINEAR MIXING SIGNALS
}

\author{
Usama Sayed Mohammed \\ Department of Electrical Engineering, Faculty of Engineering, Assiut University, \\ Assiut, Egypt E-mail: Usama@aun.edu.eg \\ Hany Saber \\ Department of Electrical Engineering, Faculty of Eng., South Valley University, \\ Aswan, Egypt E-mail: hany@svu.edu.eg
}

(Received December 20, 2008 Accepted May 9, 2009)

\begin{abstract}
In this paper, a new technique to solve the nonlinear blind source separation problem (NBSS) is introduced. The method is based on the concept of reducing the high frequency component of the nonlinear mixed signal by dividing the mixed signal into blocks in the time domain, with any arbitrary size. To remove the distortion of the nonlinear function, the discreet cosine transform (DCT) is applied on each block. By adaptively adjusting the size of the DCT block of data, the highly correlated subblocks, can be estimated, then the correlation between the highly correlated sub-blocks can be reduced. To complete the separation process, the linear blind source separation (BSS) algorithm based on the wavelet transform is used to reduced the correlation between the highly correlated DCT subblock. Performed computer simulations have shown the effectiveness of the idea, even in presence of strong nonlinearities and synthetic mixture of real world data (like speech and image signals).
\end{abstract}

KEYWORDS: nonlinear blind source separation, discreet casein transform, linearization, post nonlinear mixing and independent component analysis (ICA).

\section{INTRODUCTION}

The problem of blind source separation (BSS) consists on the recovery of independent sources from their mixture. This is important in several applications like speech enhancement, telecommunication, biomedical signal processing, etc. Most of the work on BSS mainly addresses the cases of instantaneous linear mixture [1-5]. Let $\mathbf{A}$ a real or complex rectangular $(n \times m ; n \geq m)$ matrix, the data model for linear mixture can be expressed as

$$
\mathrm{X}(\mathrm{t})=\mathrm{AS}(\mathrm{t})
$$

Where $S(t)$ represents the statistically independent sources array while $X(t)$ is the array containing the observed signals. For real world situation, however, the basic linear mixing model in equation (1) is too simple for describing the observed data. In many applications such as the nonlinear characteristic introduced by preamplifiers of receiving sensors, we can consider a nonlinear mixing. So a nonlinear mixing is more realistic and accurate than linear model. For instantaneous mixtures, a general nonlinear data model can have the form 


$$
\mathrm{X}(\mathrm{t})=f(\mathrm{~S}(\mathrm{t}))
$$

Where $f$ is an unknown vector of real functions.

The linear instantaneous mixing (BSS) can be solved using the independent component analysis (ICA). The goal of the ICA is to separated the signals by finding independent component from the data signal. The ICA is commonly used in solving the problem of the linear mixing but in the nonlinear case the ICA is not used to solve this problem. It is important to note that if $x$ and $y$ are two independent random variables, any of their functions $f(x)$ and $f(y)$ are also independent. An even more serious problem is that in the nonlinear case, $x$ and $y$ can be mixed and still be statistically independent. Several authors [6-10] have addressed the important issues on the existence and uniqueness of solutions for the nonlinear ICA and BSS problems. In general, the ICA is not a strong enough constraint for ensuring separation in the nonlinear mixing case. There are several known methods that try to solve this nonlinear BSS problem. They can roughly be divided into algorithms with a parametric approach and algorithms with nonlinear expansion approach. With the parametric model the nonlinearity of the mixture is estimated by parameterized nonlinearities. In [8] and [11], neural network is used to solved this problem. In the nonlinear expansion approach the observed mixture is mapped into a high dimensional feature space and afterwards a linear method is applied to the expanded data. A common technique to turn a nonlinear problem into a linear one is introduced in [12].

\subsection{Nonlinear Mixture Model}

A generic nonlinear mixture model for blind source separation can be described as follows:

$$
X(t)=A f(S(t)
$$

Where $S(t)$ represents the statistically independent sources array while $X(t)$ is the array containing the observed signals and $\mathrm{f}$ is unknown multiple-input and multiple-output (MIMO) mapping which called the nonlinear mixing transform (NMT). In order for the mapping to be invertible we assume that the nonlinear mapping is monotone. We make the assumption here, for simplicity and convenience, that the dimensions of $\mathrm{X}$ and $\mathrm{S}$ is equal. Fig. 1 shows the general model of the nonlinear mixing model system described in eq. (3), which contains both channel and cross-channel nonlinearities.

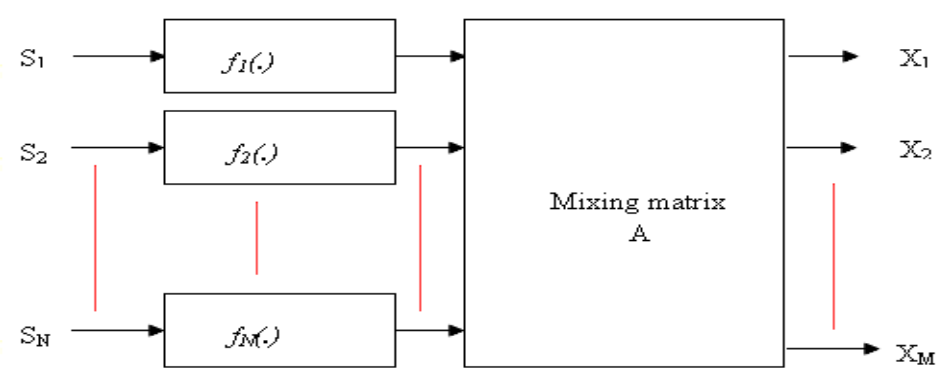

Fig.1 Nonlinear mixing model

An important special case of the nonlinear mixture is the so-called post-nonlinear (PNL) mixture, 


$$
\mathrm{X}(\mathrm{t})=f(\mathrm{AS}(\mathrm{t}))
$$

where $f$ is an invertible nonlinear function that operates componentwise and $\mathbf{A}$ is a linear mixing matrix, more detailed:

$$
X_{i}(t)=f_{i}\left(\sum_{j=1}^{m} a_{i j} S_{j}(t)\right), i=1, \ldots \ldots n
$$

Where $a_{i j}$ are the elements of the mixing matrix A. The PNL model was introduced by Taleb and Jutten[14]. It represents an important subclass of the general nonlinear model and has therefore attracted the interest of several researchers [15-19]. Applications are found, for example, in the fields of telecommunications, where power efficient wireless communication devices with nonlinear class $\mathrm{C}$ amplifiers are used [20] or in the field of biomedical data recording, where sensors can have nonlinear characteristics [21].

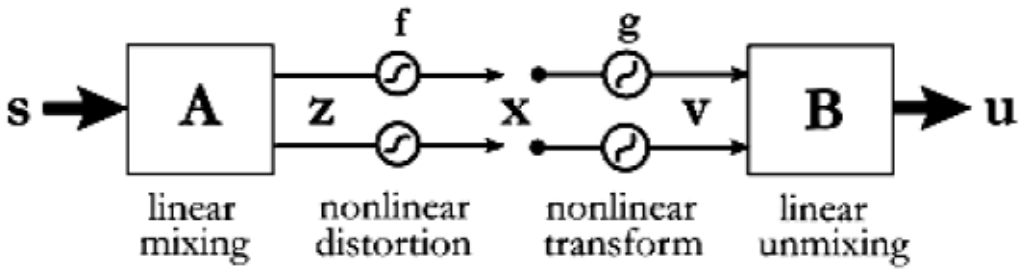

Fig.2 Building blocks of PNL mixing model and separation

In this work, a decoupled two-stage process to solve the PNL-BSS problem or the general nonlinear BSS is proposed. First, making linearization for each data vector to overcome the nonlinear function $f$. The second step is the linear BSS techniques. It is based on the time-frequency analysis to recover the underlying source signals.

\subsection{Discrete Cosine Transforms (DCT)}

Discrete cosine transform (DCT) expresses a sequence of finite data points in terms of a sum of cosine functions oscillating at different frequencies. DCT is important to numerous applications in science and engineering. In particular, a DCT is a Fourierrelated transform similar to the discrete Fourier transform (DFT), but using only real numbers. DCT are equivalent to DFT of roughly twice the length, operating on real data with even symmetry (since the Fourier transform of a real and even function is real and even), where in some variants the input and/or output data are shifted by half a sample. In DCT most of the signal information tends to be concentrated in a few lowfrequency components of the DCT. Forward DCT (N-point DCT) can be described as follows:

$$
\begin{aligned}
& X(k)=\frac{2}{N} c_{k} \sum_{n=O}^{N-1} x(n) \operatorname{co}\left[\frac{(2 n+1) k \pi}{2 N}\right] \\
& k=0,1, \ldots \ldots \ldots \ldots \ldots \ldots \ldots \ldots \ldots \ldots \ldots \ldots \ldots \ldots
\end{aligned}
$$

Where the inverse DCT (N-point IDCT) can be described as follows: 


$$
\begin{aligned}
& x(n)=\sum_{k=O}^{N-1} c_{k} X(k) \operatorname{Cos}\left[\frac{(2 n+1) k \pi}{2 N}\right] \\
& n=O, 1, \ldots \ldots \ldots \ldots \ldots \ldots . . . . .1 \\
& \text { where } c_{k}=\left\{\begin{array}{cc}
\frac{1}{\sqrt{2}} & k=O \\
1 & k \neq O
\end{array}\right.
\end{aligned}
$$

\subsection{Assumptions and Definitions}

In the proposed algorithm, there are some assumptions are considered as follows:

(1) the source signals must be independent, (2) the number of mixed signals are greater than or equal to the number of the source signals, (3) there are at most one source signal have Gaussian distribution, (4) the nonlinear function $f$ is invertible function, and (4) the mixed matrix must be of full rank.

\section{THE PROPOSED SEPARATION METHOD}

For any periodic signal, the most significant power of the DCT coefficients is concentrated in the low-frequency components. Even with the applying a nonlinear function on this signal, the distribution of the DCT coefficients will be changed keeping the most significant power in the low frequency components. After applying the DCT transform on the blocks of the mixed signals, the effect of nonlinear function can be removed by finding the highly correlated subblocks (in the low frequency components of the DCT coefficients ) and reducing this correlation. The correlation reduction process among subblocks can be done by applying a linear blind source separation technique on the correlated subblocks of the signal.

In this work, the idea of the proposed algorithm is to make linearization of the nonlinear mixed data by apply the DCT transform on the blocks of the nonlinear mixed signals and adaptively select the suitable data block size that produce highly correlated DCT coefficients and reducing this correlation. The proposed algorithm can be used with the nonlinear blind source separation problem described by eq. (3) or even for the post nonlinear form of BSS described by eq. (5). The proposed method needs the signal to be stationery. So, the mixed signals are divided, in the time domain, into small block to overcome this problem.

The proposed algorithm can be summarized as follows: (1) dividing the nonlinear mixed data in the time domain into blocks, (2) Apply the DCT transform on each block, (3) select the low frequency components of the DCT data block as a subblocks, (4) test the correlation among these subblocks with a predefined threshold (in this work the threshold of the correlation is selected to be 0.8), (5) if the correlation value is not greater than or equal the threshold, then increase the size of the subblocks and repeat step 4, (6) the correlated DCT subblocks are pre-whitened using eigenvalue decomposition of the zero time-lag correlation matrix (the basics of the pre-whitened procedure is reported in appendix A.1), (7) n-scale wavelet packet transform of the whitened subblocks resulting in n-subband signals, (8) evaluate the covariance matrix among the corresponding subband to test the correlation in the wavelet domain, (9) the joint approximate diagonalization criteria is used to minimize the zero time lag 
correlation among the subband (the joint approximate diagonalization criteria is reported in appendix A.2) [22], (10) for $\mathrm{n}$ separation matrices $\mathrm{B}$ from each subband of n-stage are estimated, using each two matrices to compute and test the global matrices "G" in eq.(8)[23], (11) using eq.(9) to test the performance of the estimated separation matrices (12) If the subband signals are correlated then the signals must be decomposed another time using the wavelet packet transform and using the minimum correlated band (MCB) search method to estimate the global separating matrix (MCB is reported in Appendix A.3). (a good measuring of the uncorrelated subbands is the performance index (PI) in eq.(9)).

$$
\begin{aligned}
& G_{i j}=B_{i} * B^{-1}{ }_{j}, \text { where } \mathrm{i} \neq \mathrm{j} \\
& \mathrm{PI}=\frac{1}{n(n-1)} \sum_{i=1}^{n} \sum_{j=1}^{n}\left(\frac{[G]_{i j}}{\max _{j}[G]_{i j}}-\mathbf{1}\right)
\end{aligned}
$$

Where $[\mathrm{G}]_{i j}$ is the $(i, j)$-element of the estimating matrix G. The smaller PI implies usually better performance in separation.

(13) using the estimated matrix which corresponding to the minimum correlated band as a separation matrix ,(14) compare the Kurtosis of the estimated signals with a predefined threshold (in this work the threshold absolute value of the Kurtosis is selected to be 4, (15) if the Kurtosis of the estimating signals is greater than the threshold, then increase the time domain block size in step (1) and repeat the steps starting from step (2).

\section{SIMULATION RESULTS AND IMPLEMENTATION}

Several types of signals with the two mixture models are used to evaluate the performance of the proposed technique. In the first example, AM modulated signal and sine wave signal is mixed by cubic function, introduced in [13], to compare the performance of the proposed algorithm with the results of BSNN (B-Spline Neural Network). In the $2^{\text {nd }}$ example and the $4^{\text {th }}$ example, the performance of the proposed algorithm in separation of real audio application is introduced. In the third example, a sinusoidal signal is mixed with white Gaussian noise to illustrate the separation of the noise from the signal. The performance of the proposed technique in separation of the nonlinear mixing of two images is introduced in the $5^{\text {th }}$ example. In all the simulation results the initial block size is selected to be 10 -sample and is adaptively increased to the proper size (highly correlation case). In the simulation results, the scatter plot is used as a tool to measurement the joint distributions [24]. Scatter plots are often used to depict joint distributions of random variables. As mentioned in Section 1.3, our basically assumption is that the original data are uncorrelated and the nonlinear mapping is monotone.

Experiment 1 (post nonlinear mixing model): Consider a twochannel nonlinear mixture with cubic nonlinearity. For the sake of simplicity, we introduce our examples for the $2 \times 2$ case i.e. considering the two-dimensional postnonlinear mixing model. 


$$
\left[\begin{array}{l}
x_{1} \\
x_{2}
\end{array}\right]=B\left[\begin{array}{l}
(.)^{3} \\
(.)^{3}
\end{array}\right]\left(A\left[\begin{array}{l}
s_{1} \\
s_{2}
\end{array}\right]\right)
$$

Where the matrices $\mathrm{B}$ and $\mathrm{A}$ are defined as

$$
B=\left[\begin{array}{cc}
.25 & .86 \\
-.86 & .25
\end{array}\right] \quad \mathrm{A}=\left[\begin{array}{cc}
.5 & .9 \\
-.9 & .5
\end{array}\right]
$$

The source vectors $S(t)$ is given by an amplitude-modulated signal and a sinusoidal signal as follows

$$
S(t)=[(0.5+\sin (6 \pi t)) \cos (100 \pi t) ; \sin (20 \pi t)]^{T}
$$

Figure 3 shows the original and the estimating sources.

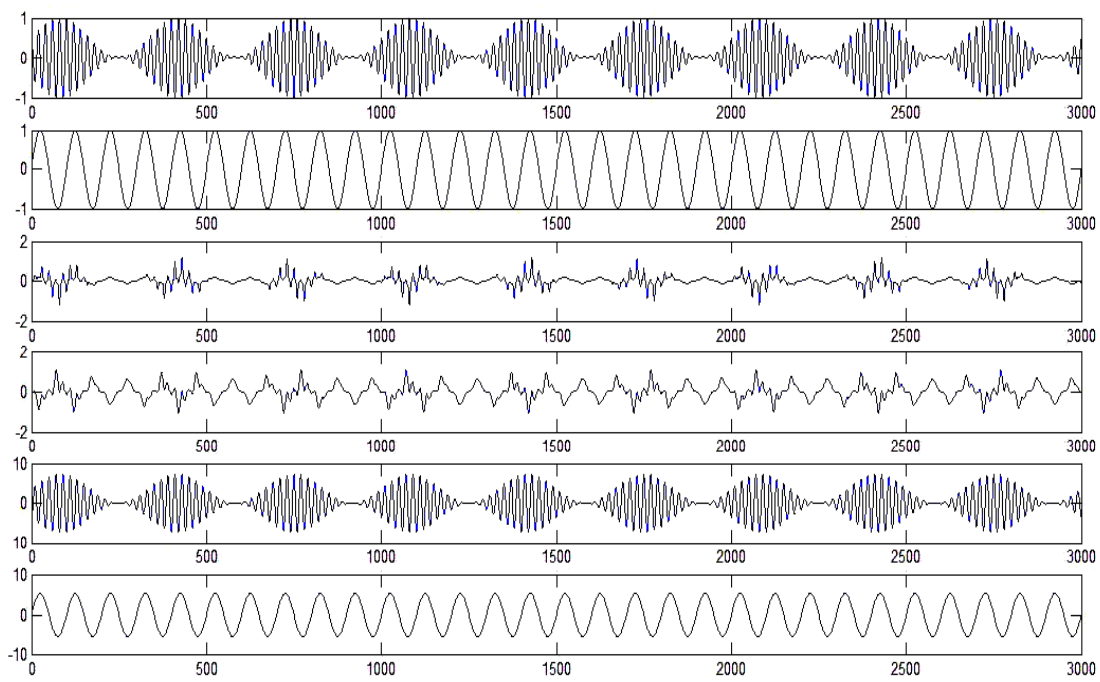

Fig. 3 the original data are the first two signals on the top ,the mixing data are in the middle and the recovering data are in the last two rows.

Experiment 2 (general nonlinear mixing model): The proposed algorithm is applied on speech signal with length of 30000 samples mixed by the following strong nonlinear function. Figure 4 shows the original and the estimating sources. Moreover, the scattering plot of the original signals, the mixed signals and the estimating signals are shown in Figure 5.

$$
\begin{aligned}
& X_{1}(t)=S_{1}(t)+S_{2}(t)^{3} \\
& X_{2}(t)=S_{1}(t)^{3}+\tanh \left(S_{2}(t)\right)
\end{aligned}
$$



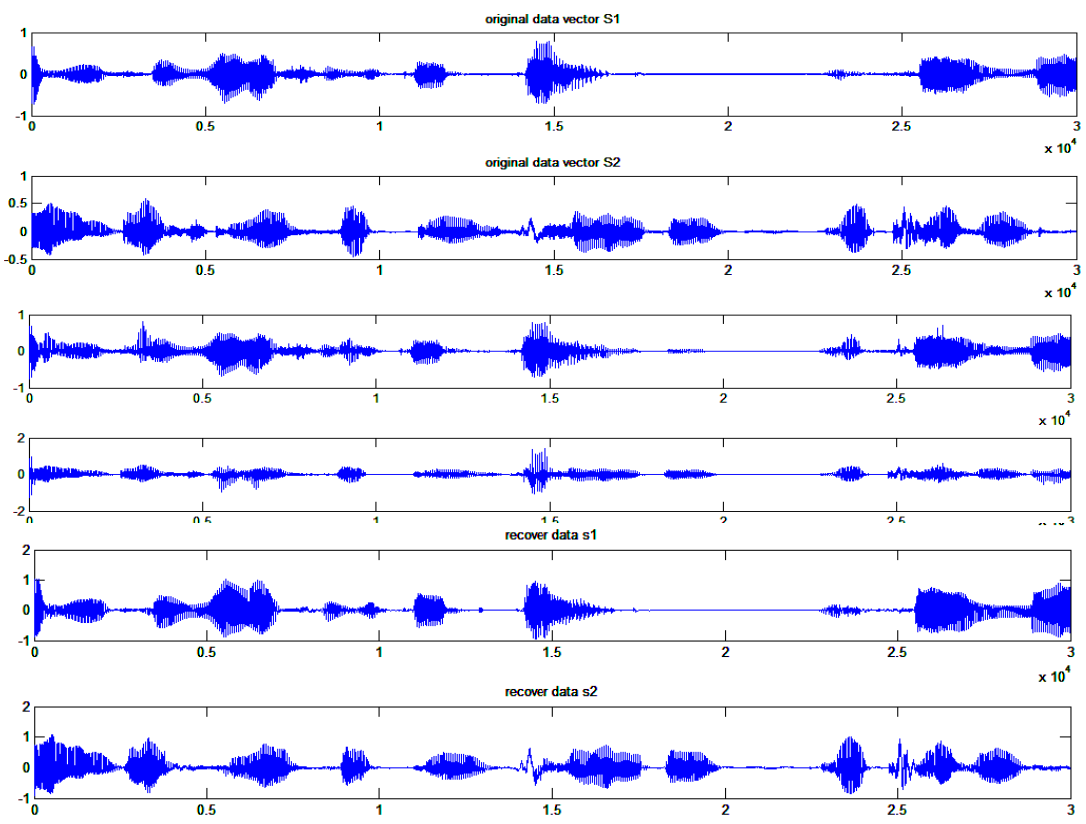

Fig. 4 the original data are the first two signals on the top ,the mixing data are in the middle and the recovering data are in the last two rows.

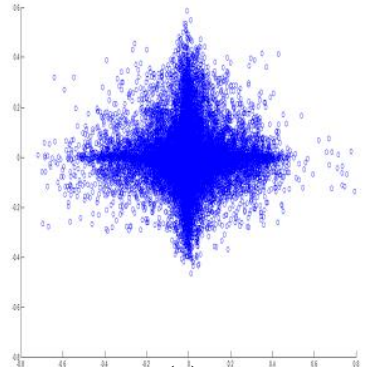

(a)

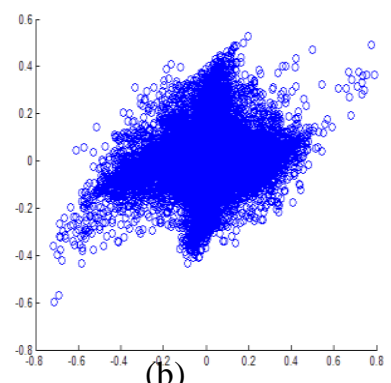

(b)

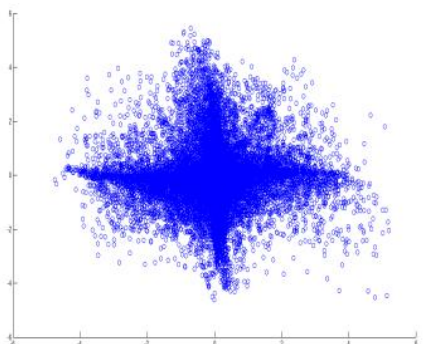

(c)

Fig.5. (a) scatter plot of the original data. (b) scatter plot of the mixed data . (c) scatter plot of the estimated data

Experiment 3 (general nonlinear mixing model): The proposed algorithm is applied on sinusoidal signal with length of 10000 samples mixed with white Gaussian noise with zero mean and $10 \%$ variance using the following strong nonlinear function. Figure 6 shows the original, the mixing and the estimating sources. Moreover, the scattering plot of the original signals, the mixed signals and the estimating signals are shown in Figure 7.

$$
\begin{aligned}
& X_{1}(t)=4 S_{1}(t)^{3}+S_{2}(t) \\
& X_{2}(t)=e^{\left(S_{1}(t)\right)}+\tanh \left(S_{2}(t)\right)
\end{aligned}
$$



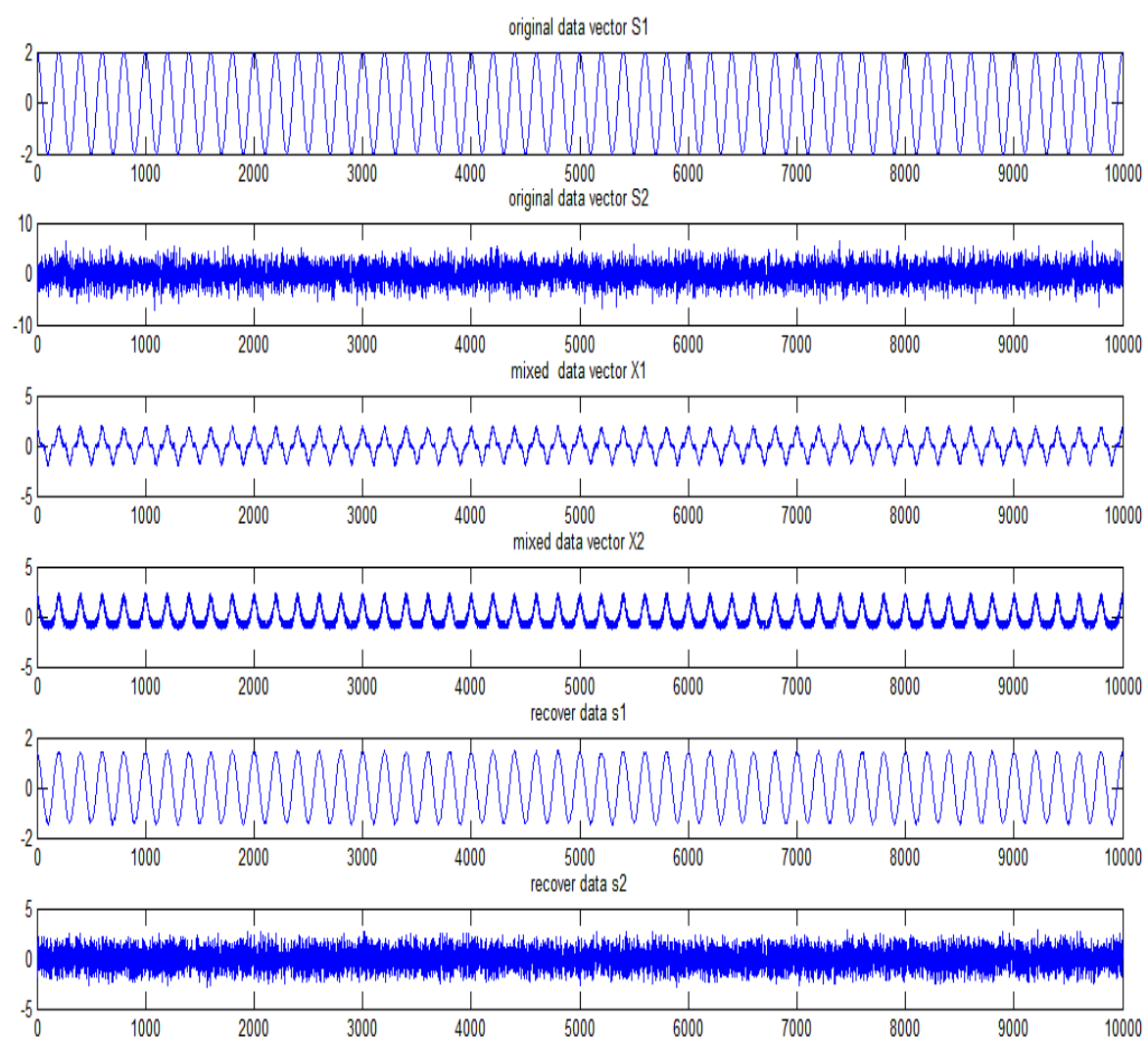

Fig. 6 the original data are the first two signals on the top ,the mixing data are in the middle and the recovering data are in the last two rows.

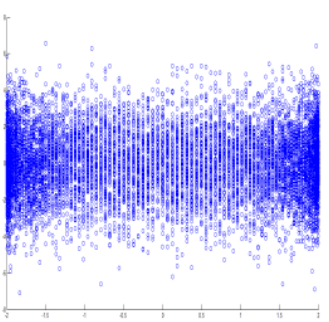

(a)

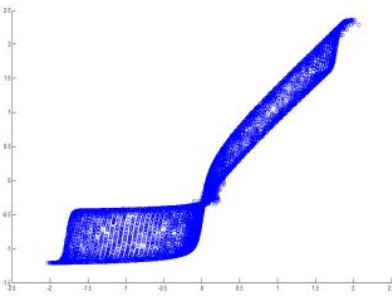

(b)

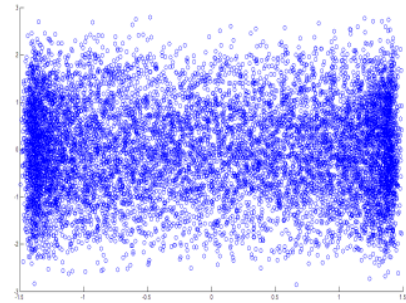

(c)

Fig.7. (a) scatter plot of the original data. (b) scatter plot of the mixed data . (c) scatter plot of the estimated data

Experiment 4 (general nonlinear mixing model): The proposed algorithm is applied on speech signal, in the experiment 2, mixed by the following strong nonlinear function. Figure 8 shows the original, the mixing and the estimating sources. Moreover, the scattering plot of the original signals, the mixed signals and the estimating signals are shown in Figure 9. 


$$
\begin{aligned}
& X_{1}(t)=\cos \left(S_{1}(t)\right) \\
& X_{2}(t)=\left(0.2 S_{1}(t)+6\right) \cos \left(S_{2}(t)\right)
\end{aligned}
$$
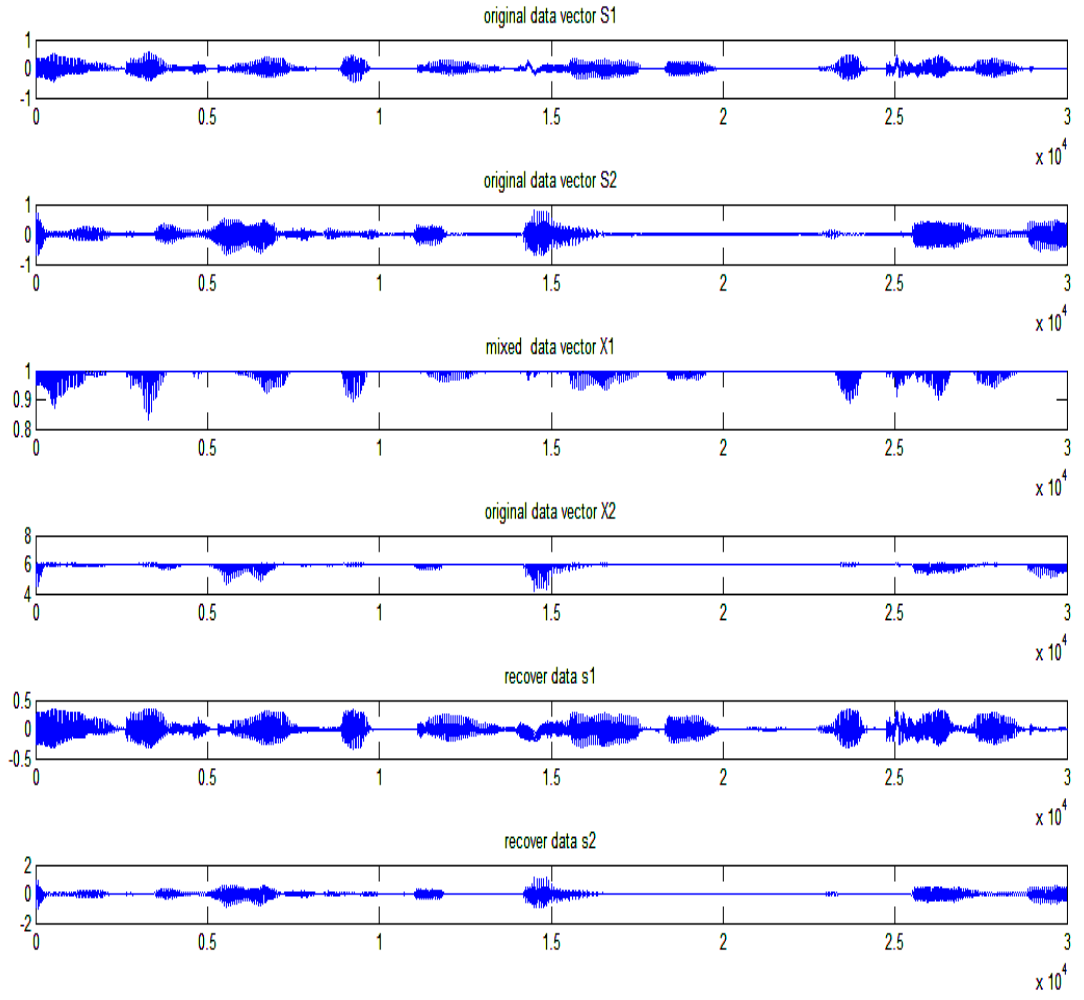

Fig. 8 the original data are the first two signals on the top the mixing data are in the middle and the recovering data are in the last two rows.

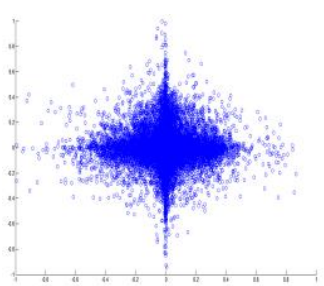

(a)

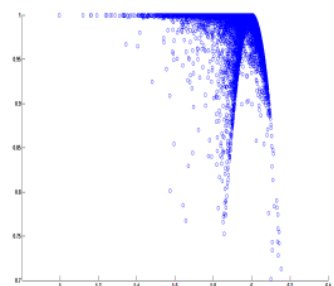

(b)

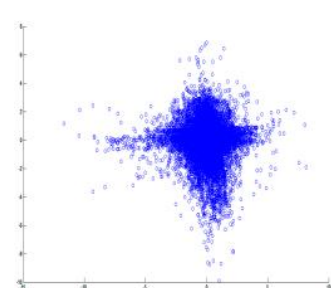

(c)

Fig.9 (a) scatter plot of the original data. (b) scatter plot of the mixed data . (c) scatter plot of the estimated data

Experiment 5 (general nonlinear mixing model): The proposed algorithm is applied on two mixed image signals. The image signals are mixed by the following strong nonlinear function. Figure 8 shows the original, the mixing and the estimating images.

$$
\begin{aligned}
& X_{1}(t)=S_{1}(t)\left(\tanh {S_{2}}_{2}(t)\right)+0.2 \sin \left(S_{2}(t)\right) \\
& X_{2}(t)=S_{2}(t)\left(\tanh \oint_{1}(t)\right)+0.2 \sin \left(S_{1}(t)\right)
\end{aligned}
$$



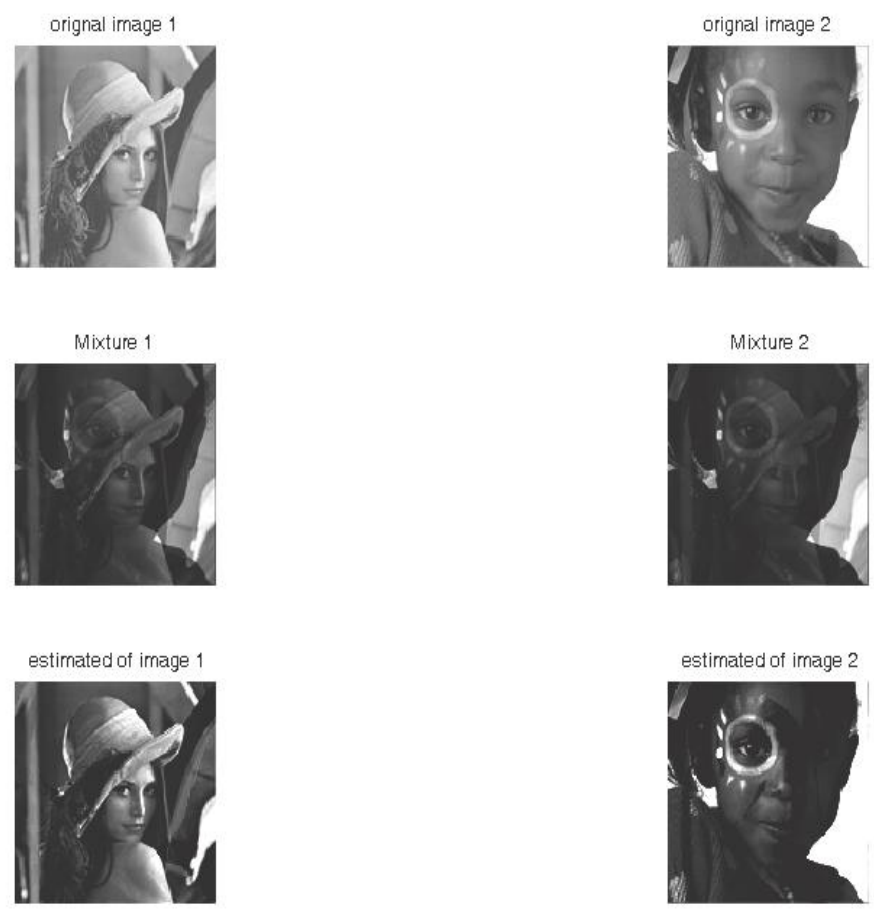

Fig. 10 the original images are the first two images on the top the mixing images are in the middle and the recovering images are in the last two rows.

The simulation result confirms the robustness of the algorithm versus the nonlinear mixing function. The the proposed algorithm is not simple and the execution computational time of the algorithm is relatively high but the performance of this algorithm is promising with all type of the nonlinear mixing model. Therefore, our future investigations will focus on reduction of the algorithm complexity.

\section{CONCLUSIONS AND DISCUSSION}

We have introduced a new adaptive algorithm to solve the problem of the nonlinear blind source separation. The algorithm depends on minimizing the correlation between the block of the data in the DCT domain. By choosing the suitable block size in the time domain and the DCT domain we can overcame the nonlinearity of the mixing function. Then a linear blind source separation algorithm is applied on the data. Wavelet based blind signal separation approach is used for blind signal separation of the DCT data block. Although the proposed algorithm is not simple but there is no restriction on the mixed model (general nonlinear or post nonlinear mixing model). Our extensive experiments have confirmed that, the use of the proposed procedure provides promising results. 


\section{APPENDIX}

\section{A.1 Spatial whitening}

The role of whitening is ideally to convert the noise-free signal vector $\mathrm{x}(\mathrm{t})$ into a new signal vector so that the zero time-lag correlation matrix of the new vector is the identity matrix. In the following analysis, the signal vector $x^{\prime}(t)$ is the DCT coefficients of the data blocks.

When the sources $s(t)$ is uncorrelated, then,

$$
\mathrm{E}\left\{\mathrm{s}_{\mathrm{i}}(\mathrm{t}) \mathrm{s}_{\mathrm{j}}(\mathrm{t})\right\}=0 \quad \text { for } \mathrm{i} \neq \mathrm{j}
$$

This condition is weaker than spatial independence, and it also can include nearly Gaussian-distributed sources. In addition to this condition, under the assumption that each source signal has unit variance, i.e.;

$$
\mathrm{R}_{\mathrm{ss}}(0)=\mathrm{E}\left\{\mathrm{s}(\mathrm{t}) \mathrm{s}^{\mathrm{T}}(\mathrm{t})\right\}=\mathrm{I}
$$

Since the DCT output can be modeled as a linear combination of the subblock components i.e. the mixing matrix A is transferred to the DCT domain. Consider the corresponding correlation matrix of the input (DCT) Data

$$
\begin{aligned}
\mathrm{R}_{\mathrm{xx}}(0) & =\mathrm{E}\left\{\mathrm{x}^{\prime}(\mathrm{t}) \mathrm{x}^{\prime \mathrm{T}}(\mathrm{t})\right\} \\
& \approx \mathrm{AE}\left\{\mathrm{s}(\mathrm{t}) \mathrm{s}^{\mathrm{T}}(\mathrm{t})\right\} \mathrm{A}^{\mathrm{T}} \\
& =\mathrm{AR}_{\mathrm{ss}}(0) \mathrm{A}^{\mathrm{T}}
\end{aligned}
$$

The mixing matrix A can be parameterized as QDG with $\mathrm{Q}$ and $\mathrm{G}$ is unitary matrices and $\mathrm{D}$ is a diagonal matrix. The correlation matrix of the observed signal is given by

$$
\mathrm{R}_{\mathrm{xx}}=\mathrm{AR}_{\mathrm{ss}} \mathrm{A}^{\mathrm{T}}=\mathrm{AA}^{\mathrm{T}}=\mathrm{QD}^{2} \mathrm{Q}^{\mathrm{T}}
$$

The eigenvalue decomposition $(E V D)$ of $R_{x x}(0)$, which is unique, can be written as;

$$
\mathrm{R}_{\mathrm{xx}}(0)=\mathrm{vkv} \mathrm{T}^{\mathrm{T}}
$$

With $\mathrm{v}$ is a unitary matrix and $\mathrm{k}$ is a diagonal matrix. By identification, it is found that; $\mathrm{v}=\mathrm{Q}$ and $\mathrm{k}=\mathrm{D}^{2}$.

Then, the whitening matrix $\mathrm{w}$ is defined as $\mathrm{w}=\mathrm{k}^{-0.5} \mathrm{v}^{\mathrm{T}}$ and the whitened signals are defined as:

$$
\mathrm{z}(\mathrm{t})=\mathrm{w} \mathrm{x}^{\prime}(\mathrm{t}) \approx \mathrm{wA} \mathrm{s}(\mathrm{t})=\mathrm{u} \mathrm{s}(\mathrm{t})
$$

and the signal in the transform domain can be written as:

$$
\mathrm{Z}(\mathrm{k})=\mathrm{w} \mathrm{X}(\mathrm{k})=\mathrm{w} \text { AS }(\mathrm{k})=\mathrm{u} \mathrm{S}(\mathrm{k})
$$

In this work, the unitary matrix $\mathrm{u}$ will be estimated in the wavelet domain to perform separation. From each corresponding subband in the wavelet domain, the covariance matrix is estimated to construct a set of covariance matrices. Then, the joint approximate diagonalization (JAD) process is applied on this set to obtain on the unitary matrix $\mathrm{u}$, and the separating matrix will be as follows:

$$
\mathrm{B}=\mathrm{u}^{\mathrm{T}} \mathrm{w}
$$




\section{A.2 Joint Approximate Diagonalization (JAD)}

The approximate joint diagonalization of a combined set of arbitrary matrices, the "off" of an $\mathrm{n} \times \mathrm{n}$ matrix $\mathrm{M}$ with entries $\mathrm{M}_{i j}$, can be defined as

$$
\operatorname{Off}(M)=\sum_{1 \leq i \neq j \leq n}|M i j|^{2}
$$

and the unitary diagonalization of a matrix $\mathrm{M}$ is equivalent to zeroing $\mathrm{Off}\left(\mathrm{V}^{T} \mathrm{MV}\right)$ by some unitary matrix $\mathrm{P}$. The spectral theorem states that only normal matrices can be unitarily diagonalized. In addition, if a matrix $\mathrm{M}$ is in the form $\mathrm{M}=\mathrm{UDU}^{\mathrm{T}}$, where $\mathrm{U}$ is unitary and $\mathrm{D}$ is diagonal with distinct diagonal elements, then it may be unitarily diagonalized only by unitary matrices that are essentially equal to $\mathrm{U}$, that is, if Off $\left(\mathrm{P}^{\mathrm{T}} \mathrm{MP}\right)=0$, then $\mathrm{P}=\mathrm{U}$.

Consider a set $\mu=\left\{\mathrm{M}_{\mathrm{j}}, \ldots, \mathrm{M}_{\mathrm{k}}\right\}$ of $k$ matrices of size $\mathrm{n} \times \mathrm{n}$. the joint diagonalization (JD) criterion is defined, for any $n \times n$ matrix $P$, as the following nonnegative function of $\mathrm{P}$ :

$$
\mathrm{C}(\mu, P)=\sum_{k=1, k} \text { Off }\left\{P^{T} M_{K} P\right\}
$$

A unitary matrix is said to be a joint diagonalizer of the set $\mu$ if it minimizes the JD criterion over the set of all unitary matrices.

Let us first consider the case where each matrix in the set $\mu$ is in the form $\mathrm{M}_{\mathrm{k}}=\mathrm{UD}_{\mathrm{k}} \mathrm{U}^{\mathrm{T}}$, where $\mathrm{D}_{\mathrm{k}}$ is a diagonal matrix. Then clearly, $\mathrm{C}(\mu, U)=0$, and this is the global minimum of the JD criterion since $\mathrm{C}(\mu, P) \geq 0$ for any matrix $P$. Thus, if each matrix in the set $\mu$ can be unitarily diagonalized by $\mathrm{U}$, then according to our definition, matrix $U$ is a joint diagonalizer of $\mu$.

\section{A.3 Minimum correlated band (MCB) search technique}

The wavelet transform of the DCT coefficients may be correlated in subband. We need that at least two bands is uncorrelated to achieve the separation. To blindly detect these uncorrelated bands in the $n$-stage, a set of separating matrices: $\mathrm{B}_{1}, \mathrm{~B}_{2}, \mathrm{~B}_{3}, \ldots \mathrm{B}_{\mathrm{m}}$ from each two bands in the m-band in the wavelet domain are used to compute the global matrices [23] as:

$\mathrm{G}_{i j}=\mathrm{B}_{i} * \mathrm{~B}_{j}{ }^{\neq}$, for $i \neq j$

For all possible pairs $i$ and $j$, where $\mathrm{B}_{j}$ is the estimated separating matrix for the $\mathrm{j}$-th frequency subband and $\mathrm{Bj}^{\neq}$is its pseudo-inverse which is equal to the separating

matrix. If the specific sub-components of interest are uncorrelated for at least two subbands, or more generally two subsets of multi-band, say for the subband " $p$ " and subband " $q$ ", then the global matrix will be as follows:

$$
\mathrm{G}_{p q}=\mathrm{B}_{p} * \mathrm{~B}_{q}{ }^{\neq} \approx \mathrm{P}
$$

Will be a sparse generalized permutation matrix $\mathrm{P}$ with special structure with only one non-zero (or strongly dominating) element in each row and each column. 


\section{REFERENCES}

[1] Amari, A. Cichocki, and H. H. Yang. "A new learning algorithm for blind signal separation." In NIPS 95, pages 882-893. MIT Press, 1996.

[2] Bell and T. Sejnowski. " information-maximization approach to blind separation and blind deconvolution" Neural Computation, vol.7:1129-1159, 1995.

[3] Cardoso. "infomax and maximum likelihood for source separation" IEEE Letters on Signal Processing, 4:112-114, 1997.

[4] W. Lee, M. Girolami, and T. Sejnowski. "Independent component analysis using an extended infomax algorithm for mixed sub-Gaussian and super-Gaussian sources." Neural Computation, 11: 417-441, 1999.

[5] D.-T. Pham, and J.-F. Cardoso, "Blind separation of instantaneous mixtures of nonstationary sources", IEEE Trans. on Signal Processing, 49(9): 1837-1848, Sep. 2001.

[6] C. Jutten and A. Taleb, "Source separation: from dusk till dawn," in Proc. 2nd Int. Workshop on Independent Component Analysis and Blind Source Separation (ICA2000), pages 15-26, Helsinki, Finland, 2000.

[7] A. Hyv"arinen and P. Pajunen, "Nonlinear independent component analysis: Existence and uniqueness results," Neural Networks, 12(3): 429-439, 1999.

[8] A. Taleb and C. Jutten, "Source separation in post-nonlinear mixtures," IEEE Trans. on Signal Processing, 47(10): 2807-2820, 1999.

[9] A. Taleb, "A generic framework for blind source separation in structured nonlinear models," IEEE Trans. on Signal Processing, 50(8): 1819-1830, 2002.

[10] J. Eriksson and V. Koivunen, "Blind identifiability of class of nonlinear instantaneous ICA models," in Proc. of the XI European Signal Proc. Conf. (EUSIPCO 2002), vol. 2, Toulouse, France, , pages 7-10, September 2002.

[11] Luis B. Almeida, "Linear and nonlinear ICA based on mutual information " the MISEP method, Signal Processing, 84(2):231-245. Special Issue on Independent Component Analysis and Beyond 2004.

[12] Tobias Blaschke and Laurenz Wiskott . "Independent Slow Feature Analysis and Nonlinear Blind Source Separation "Proc. of the 5th Intl. Conf. on Independent Component Analysis and Blind Signal Separation Granada, Spain, pages 22-24 September 2004

[13] Mirko Solazzi and Aurelio Uncini, " Spline Neural Networks for Blind Separation of Post-Nonlinear-Linear Mixtures" IEEE transactions on circuits and systems-i: regular papers, 51(4): 817-829, 2004.

[14] Taleb and C. Jutten. Nonlinear source separation: The post-nonlinear mixtures. In Proc. European Symposium on Artificial Neural Networks, pages 279-284, Bruges, Belgium, 1997.

[15] T.-W. Lee, B.U. Koehler, and R. Orglmeister. Blind source separation of nonlinear mixing models. IEEE International Workshop on Neural Networks for Signal Processing, pages 406-415, 1997.

[16] H.-H. Yang, S. Amari, and A. Cichocki. Information-theoretic approach to blind separation of sources in non-linear mixture. Signal Processing, 64(3):291-300, 1998.

[17] A. Taleb and C. Jutten. Source separation in post-nonlinear mixtures. IEEE Trans. on Signal Processing, 47(10):2807-2820, 1999. 
[18] A. Ziehe, M. Kawanabe, S. Harmeling, and K.-R. M“uller. Separation of postnonlinear mixtures using ACE and temporal decorrelation. In T.-W. Lee, editor, Proc. Int. Workshop on Independent Component Analysis and Blind Signal Separation (ICA2001), pages 433-438, San Diego, California, 2001.

[19] C. Jutten and J. Karhunen. Advances in nonlinear blind source separation. In Proc. of the 4th Int. Symp. on Independent Component Analysis and Blind Signal Separation (ICA2003), pages 245-256, Nara, Japan, Invited paper in the special session on nonlinear ICA and BSS, April 2003..

[20] L. E. Larson. "Radio frequency integrated circuit technology low-power wireless communications". IEEE Personal Communications, 5(3): 11-19, 1998.

[21] A. Ziehe, K.-R. M"uller, G. Nolte, B.-M. Mackert, and G. Curio. Artifact reduction in magnetoneurography based on time-delayed second-order correlations. IEEE Trans. Biomed. Eng., 47(1): 75-87, 2000.

[22] Jean-François Cardoso and Antoine Souloumiac." Jacobi angles for simultaneous diagonalization" Siam J. Mat. Anal. Appl., 17(1):161-164, 1996.

[23] T. Tanaka and A. Cichocki, "Subband decomposition independent component analysis and new performance criteria," in Proceedings of International Conference on Acoustics, Speech, and Signal Processing (ICASSP2004), vol. V, (Montreal, Canada), pages 541-544, May 2004.

[24] Luis B. Almeida, "Nonlinear Source Separation" Institute of telecommunication , Portugal ISBN: 1598290304 ,Morgan \& Claypool 2006.

\section{طريقه ذاتية التكيف لفصل الإشارات المبهمة والمخلوطة لاخطيا}

في هذه المقالة تم اقتراح طريقة جديدة يمكن بها حل مشكلة الخلط المبهم الغير خطى للإثارات. تم في

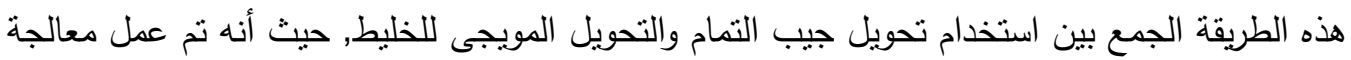
مبدئية للخليط (Linearization) باستخدام تحويل جيب التمام ثم أستخدم التحليل المويجى للخليط المعالج لإتمام عملية الفصل. الفكرة الأساسية تعتمد علي تقليل الارتباط بين معاملات الحزم المتتاظرة.

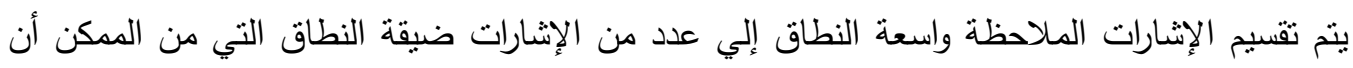

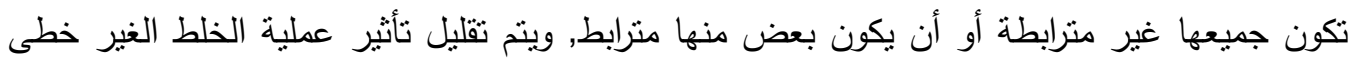

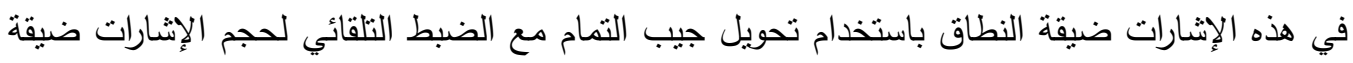

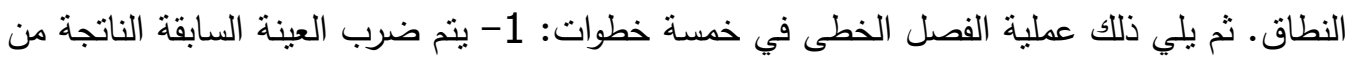
تحويل جيب التمام للإثشارات في مصفوفة التعامد لنحصل علي إثنارات متعادة. 2- باستخدام التحويل

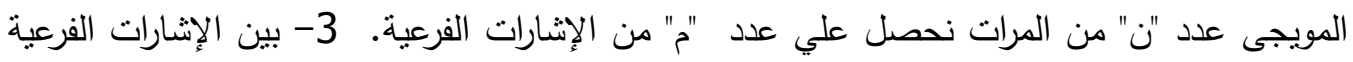
المتتاظرة في النطاق نحصل علي مصفوفة الترابط. 4-باستخدام طريقة الترابط القطري التقريبي(JAD) والتي تقلل الترابط بين النطاقات المتتاظرة نحصل علي المصفوفة المتعامدة التي تدور الإثارات 
المتعامدة بالزاوية المطلوبة 5- إذا كان هناك عدد من النطاقات مترابطة في العدد "م" من النطاقات

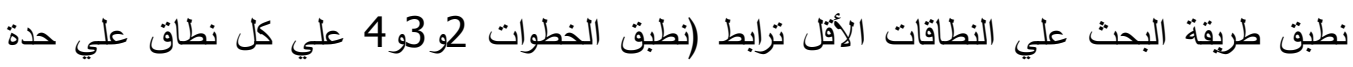

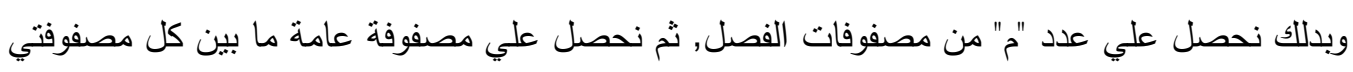
فصل, التي تختبر الترابط بين كل نطاقين ونأخذ أفضل مصفوفة عامة والأفضل هي تلأك المصفوفة

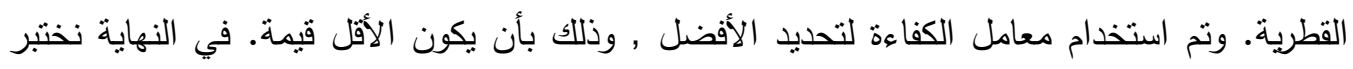

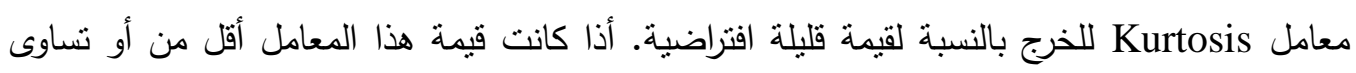
القيمة الافتراضية يتم إيقاف عملية الفصل. أذا كانت قيمة هذا المعامل أكبر من القيمة الافتراضية يتم

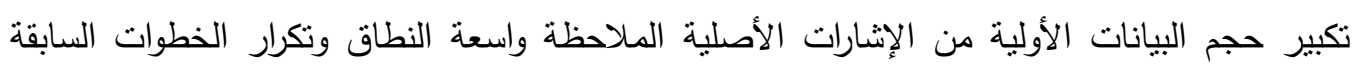

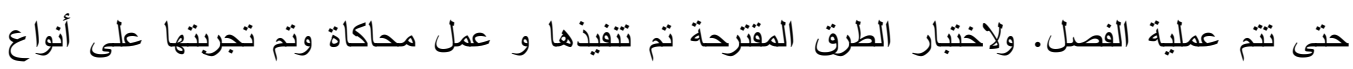
مختلفة من الإثارات (الصوت, الموسيقى,...) وعدد مختلف من مصفوفات الخلط. وقد أثنتت هذه تهذه الطريقة كفاءة عالية في عملية الفصل لهذه الإشارات مع جميع الاحتمالات الخاصة بعملية الخلط. 